\title{
The magnetic structure of neutron stars and their surface-to-core temperature relation (Research Note)
}

\author{
A. Y. Potekhin ${ }^{1,2,3}$, V. Urpin ${ }^{2,3}$, and G. Chabrier ${ }^{1}$ \\ 1 École Normale Supérieure de Lyon (CRAL, CNRS UMR 5574), 46 allée d'Italie, 69364 Lyon Cedex 07, France \\ 2 Ioffe Physico-Technical Institute, Politekhnicheskaya 26, 194021 St. Petersburg, Russia \\ e-mail: palex@astro.ioffe.ru \\ ${ }^{3}$ Isaac Newton Institute of Chile, St. Petersburg Branch, Russia
}

Received 14 June 2005 / Accepted 9 August 2005

\section{ABSTRACT}

We study the relation between the mean effective surface temperature $\bar{T}_{\mathrm{s}}$ and the internal temperature $T_{\mathrm{b}}$ for magnetic neutron stars, assuming that the magnetic field near the surface has a small-scale structure. The heavy-element (iron) and light-element (accreted) heat-blanketing envelopes are considered, and the results are compared with the case of a dipole magnetic field. We argue that the difference in the $T_{\mathrm{b}}\left(\bar{T}_{\mathrm{s}}\right)$-relation for different magnetic configurations is always much smaller than the possible difference caused by variations of the chemical composition in the envelope.

Key words. stars: neutron - stars: magnetic fields - dense matter - conduction - pulsars: general

\section{Introduction}

The most direct evidence for the nature of a poorly known superdense matter in neutron stars is likely to emerge from comparisons of surface thermal radiation measurements with predictions of neutron star cooling models (e.g., Pethick 1992; Page 1997, 1998; Yakovlev \& Pethick 2004). Modeling of neutron star cooling is a complex problem that, generally, requires calculations of the temperature profile from the surface to the core at various stages of the evolution. The temperature profile for nonmagnetic neutron stars has been the subject of study for many authors (e.g., Gudmundsson et al. 1983; Nomoto \& Tsuruta 1987; Potekhin et al. 1997, and references therein). The key issue of these studies is the so-called $T_{\mathrm{b}}\left(T_{\mathrm{s}}\right)$-relation used in cooling models ( $T_{\mathrm{s}}$ and $T_{\mathrm{b}}$ are the surface and internal temperature, respectively).

Most of the neutron stars, however, possess surface magnetic fields $B \sim 10^{12}-10^{13} \mathrm{G}$, and some neutron stars are possibly magnetars with $B \gtrsim 10^{14} \mathrm{G}$. The internal magnetic field can be even higher. Such strong fields can affect the properties of plasma in neutron stars and alter the $T_{\mathrm{b}}\left(T_{\mathrm{s}}\right)$-relation. In general, the magnetic field strength and direction varies over the stellar surface, and hence $T_{\mathrm{s}}$ may be different for different surface points. In many applications (e.g., in the neutron-star cooling theory) it is sufficient to know the mean effective surface temperature $\bar{T}_{\mathrm{s}}$ instead of the position-dependent $T_{\mathrm{s}}$. The effective temperature is defined by the Stefan law, $L=4 \pi R^{2} \sigma \bar{T}_{\mathrm{s}}^{4}$, where $\sigma$ is the Stefan-Boltzmann constant, and $L$ is the thermal luminosity in a local neutron-star reference frame, integrated over the surface. The apparent luminosity measured by a distant observer is $L^{\infty}=\left(1-r_{g} / R\right) L$, and the apparent surface temperature inferred by the observer from the radiation spectrum is $T_{\mathrm{s}}^{\infty}=T_{\mathrm{s}} \sqrt{1-r_{g} / R}$ (e.g., Thorne 1977).

The effects of a strong magnetic field on thermodynamic and kinetic properties of the outer neutron star layers have been reviewed, for instance, by Yakovlev \& Kaminker (1994) and Ventura \& Potekhin (2001). The thermal structure of magnetized neutron stars has been analyzed by a number of authors, often adopting a simplified magnetic geometry. For instance, much attention has been paid to the cases of the radial magnetic field (e.g., Hernquist 1985; Van Riper 1988; Schaaf 1990; Heyl $\&$ Hernquist 1998) or the tangential field (with the field lines parallel to the surface; e.g., Hernquist 1985; Schaaf 1990; Heyl $\&$ Hernquist 1998). The quantizing radial field field decreases the difference between $T_{\mathrm{s}}$ and $T_{\mathrm{b}}$, whereas the tangential field increases this difference.

The case of an arbitrary inclination of the field lines has been considered by Greenstein \& Hartke (1983) and Page (1995) who argued that the $T_{\mathrm{b}}\left(T_{\mathrm{s}}\right)$-relation for such a field can be constructed in a simple way from the relations for the radial and tangential magnetic fields. Numerical calculations of the thermal structure (Potekhin \& Yakovlev 2001; Potekhin et al. 2003) confirm an accuracy of this approximation. These calculations show that the $T_{\mathrm{b}}\left(\bar{T}_{\mathrm{s}}\right)$-relation for the dipole magnetic configuration is almost independent of the field for 
$B \lesssim \mathrm{a}$ few $\times 10^{13} \mathrm{G}$. The reason of such behaviour is the compensation of a decrease in the thermal flux near the magnetic equator by an increase in the flux near the pole.

This result can essentially simplify the cooling calculations for magnetized neutron stars with dipole magnetic fields. It is likely, however, that the surface magnetic field of neutron stars departs from a dipole configuration. Arons \& Scharlemann (1979) and Arons (1993, 2000) noted that pulsars with long periods require a more complex field configuration than a dipole if pair creation is essential for the mechanism of radio emission. Gil \& Mitra (2001) and Gil \& Melikidze (2002) also noted that radio emission of many pulsars can be explained if one adopts the model with a strong $\left(\gtrsim 10^{13} \mathrm{G}\right)$ and complex surface field with a small curvature of the field lines $\left(\sim 10^{5} \mathrm{~cm}\right)$. Gil \& Sendyk (2000) found that the behaviour of drifting subpulses observed in many pulsars is consistent with the vacuum gap maintained by a strong sunspot-like magnetic field. Recent observations of the X-ray spectra of some pulsars provide an opportunity to estimate the magnetic field near the neutron star surface. For example, a possible interpretation of the feature observed in the spectrum of PSR B1821-24 as a cyclotron emission (Becker et al. 2003; see, however, Mineo et al. 2004) indicates that the local magnetic fields on the neutron star surface can exceed the conventional dipole field inferred from the spin-down data.

The growing evidence for the distinction between the local field strength at the stellar surface and the global dipole field suggests that this can be a general phenomenon in neutron stars. Several theoretical explanations of this phenomenon were suggested. Ruderman (1991) considered a "plate tectonics" model, in which a complex configuration of the surface magnetic field of a neutron star is a result of neutron-star crust cracking and platelet movements. Ruderman et al. (1998) found that this model agrees with observations of pulsar glitches. Geppert et al. (2003) suggested a Hall-driven mechanism of the formation of the sunspot-like magnetic-field structure at the neutronstar surface. The presence of small scale field components can also be generated during the initial convective stage of evolution, e.g., by the turbulent mean-field dynamo (Thompson \& Duncan 1993; Bonanno et al. 2003, 2005). The unstable stage in proto-neutron stars lasts $\sim 30-40 \mathrm{~s}$, which is sufficient for a dynamo to reach a saturation level. The magnetic field generated in proto-neutron stars will be frozen into the crust that starts to form almost immediately after convection stops. Since the crustal conductivity is high, both the large and relatively small scale $\left(\sim 10^{5} \mathrm{~cm}\right)$ fields can survive during a long time comparable to the lifetime of pulsars, $\sim 10^{8} \mathrm{yr}$ (Urpin \& Gil 2004).

If the dipole field in neutron stars is accompanied by a stronger small-scale field, then the thermal structure of the surface layers can be changed qualitatively. For instance, the difference between the equator and polar temperature should be much reduced. The small-scale field can also affect the total photon flux at given $T_{\mathrm{b}}$ - that is the $T_{\mathrm{b}}\left(\bar{T}_{\mathrm{s}}\right)$ relation. In this paper, we consider the thermal structure and calculate the $T_{\mathrm{b}}\left(\bar{T}_{\mathrm{s}}\right)$ relation in the case of a neutron star with a strong small-scale magnetic field in the envelope.

\section{Statement of the problem}

The thermal structure of the neutron star envelope is considered in a steady state plane-parallel approximation. We assume that $T_{\mathrm{b}}$ is equal to the temperature at the inner boundary of the envelope (taken at the neutron drip density $4 \times 10^{11} \mathrm{~g} \mathrm{~cm}^{-3}$ ) and does not vary over this boundary.

The statement of the problem for calculations of the temperature profile in neutron star envelopes has been described in detail by Potekhin et al. (2003). In the present study, the only difference is the expression for the heat flux, that should incorporate the effect of small-scale magnetic fields. In the magnetic field $\boldsymbol{B}$, the heat flux is related to the temperature gradient by

$\boldsymbol{F}=-\kappa_{\|}(B) \nabla_{\|} T-\kappa_{\perp}(B) \nabla_{\perp} T-\kappa_{\wedge}(B) \boldsymbol{b} \times \nabla T$,

where the tensor and vector components along and across the field are marked by $\|$ and $\perp$, respectively, and $\kappa_{\wedge}$ is the socalled Hall component; $\boldsymbol{b}=\boldsymbol{B} / \boldsymbol{B}$. Generally, all components of the thermal conductivity tensor depend on the field strength, $B$. The vector components in Eq. (1) are

$\nabla_{\|} T=\boldsymbol{b} \cdot(\boldsymbol{b} \cdot \nabla T), \quad \nabla_{\perp} T=\nabla T-\boldsymbol{b} \cdot(\boldsymbol{b} \cdot \nabla T)$.

We assume that small-scale fields are stronger than the largescale field (as it follows from the turbulent dynamo models, e.g., Thompson \& Duncan 1993; Bonanno et al. 2005), and that the length-scale of a temperature variation over the surface is much larger than the vertical length-scale. This allows us to apply the local plane-parallel approximation for studying the mean thermal structure of the heat-blanketing envelope (see Gudmundsson et al. 1983 and Potekhin et al. 2003, for discussion). Then, averaging Eq. (1) over the directions of the magnetic field (that is approximately equivalent to averaging over the stellar surface), we obtain for the mean heat flux

$\langle F\rangle=\frac{1}{3}\left[\kappa_{\|}(B)+2 \kappa_{\perp}(B)\right] \frac{\mathrm{d} T}{\mathrm{~d} z}$,

where $z$ is the local proper depth in the envelope. This is equivalent to Eq. (7) of Potekhin et al. (2003), but with the effective thermal conductivity $\kappa$ replaced by its average over all angles. Thus, unlike Potekhin et al. (2003), where the variations in strength and direction of the magnetic field were assumed smooth over the stellar surface and small compared to the temperature gradient in the heat-blanketing envelope, which allowed us to treat the field as locally constant, here we consider the opposite limit of a rapidly varying field direction (i.e., "entangled" field lines). For simplicity, we assume in what follows that the average strength of small scale magnetic fields does not depend on the depth.

We calculate the thermal structure of a neutron star envelope by integrating Eq. (3) with the use of the numerical scheme developed previously (Potekhin et al. 1997, 2003). We examine models of envelopes composed of iron and accreted material. The accreted envelope is assumed to be the same as described by Potekhin et al. (1997): the surface H layer is followed by $\mathrm{He}, \mathrm{C}, \mathrm{O}$, and $\mathrm{Fe}$ layers. The physics input (equation of state, radiative opacities, and electron thermal conductivities) is described in Potekhin et al. (2003). Note some uncertainty of this input for the outermost layer of the iron envelope 

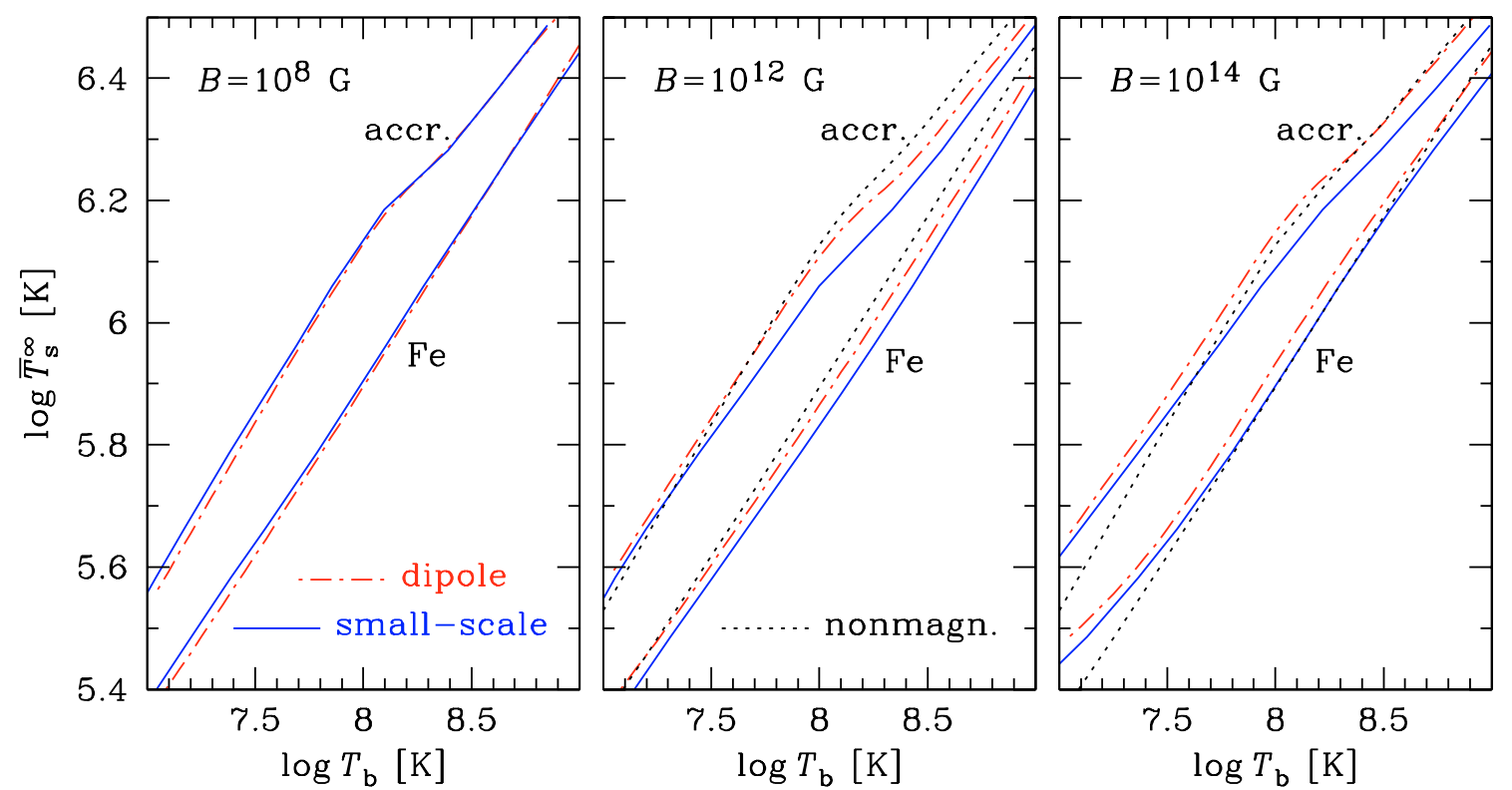

Fig. 1. The redshifted effective surface temperature versus the internal temperature for a canonical neutron star $\left(M=1.4 M_{\odot}, R=10 \mathrm{~km}\right)$ with the magnetic field $B=10^{8}, 10^{12}$, and $10^{14} \mathrm{G}$ (left, middle, and right panels) and for the case of dipole (dot-dash lines) and small-scale (solid lines) fields. The upper and lower curves represent the accreted and iron heat-blanketing envelope, respectively.

where Fe plasma is only partly ionized. In this case, the equation of state and opacities are based on the Thomas-Fermi and mean-ion approximations. For the accreted envelope, in contrast, we use the accurate equation of state and opacities for the partially ionized hydrogen plasma in a strong magnetic field (Potekhin \& Chabrier 2004, and references therein).

\section{Results}

The calculated temperature profiles and their dependences on $B$ and $T_{\mathrm{b}}$ are quite similar to those presented in Potekhin et al. (2003). Therefore we will not describe the results in detail but focus on the comparison of the $T_{\mathrm{b}}\left(\bar{T}_{\mathrm{s}}\right)$ relation for the smallscale and dipole fields.

Examples of such a comparison are shown in Figs. 1 and 2. The panels (from left to right) in Fig. 1 correspond to $B=$ $10^{8} \mathrm{G}, 10^{12} \mathrm{G}$, and $10^{14} \mathrm{G}$, typical for millisecond pulsars, ordinary radio pulsars and anomalous X-ray pulsars, respectively; $B$ is the average field strength in the case of a small-scale field and the field at the magnetic pole in the dipole field model. For convenience, the non-magnetic $T_{\mathrm{b}}\left(\bar{T}_{\mathrm{s}}\right)$-relations are shown in the middle and right panels of Fig. 1 by the dotted lines.

Figure 2 shows the dependence of $\bar{T}_{\mathrm{s}}^{\infty}$ on $B$ for different models and for two $T_{\mathrm{b}}$ values. Independent of the field geometry, a weak field $\left(B \lesssim 10^{8} \mathrm{G}\right)$ does not affect the $T_{\mathrm{b}}\left(\bar{T}_{\mathrm{s}}\right)$-relation for both the iron and accreted envelopes. With increasing $B$, the effective temperature first decreases and then increases nonmonotonically. This behaviour is explained as follows. The value of $T_{\mathrm{s}}$ at a given $T_{\mathrm{b}}$ is controlled mainly by the opacity values in the "sensitivity strip" (Gudmundsson et al. 1983) located typically at $\rho \sim 10^{4}-10^{8} \mathrm{~g} \mathrm{~cm}^{-3}$, where heat transport is provided by the electron conduction. In a strong magnetic field, the electron thermal conductivity as a function of $\rho$ undergoes oscillations when the electron Fermi

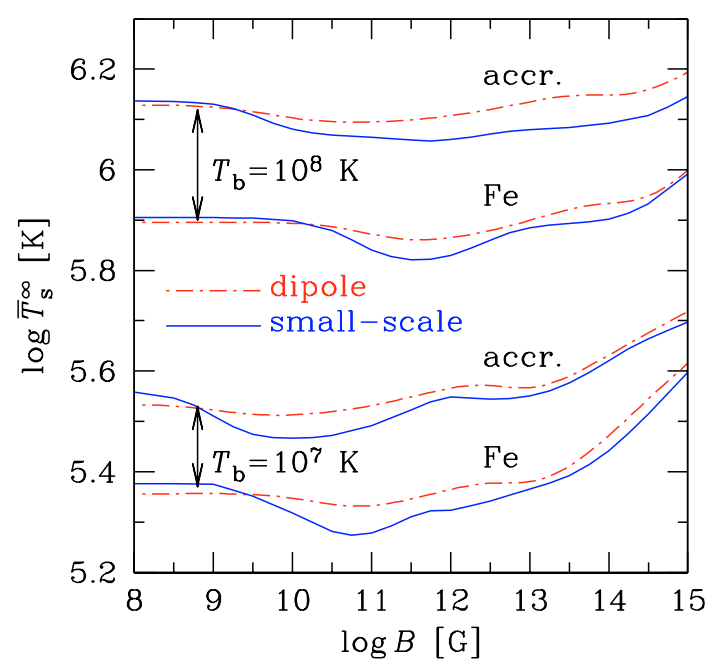

Fig. 2. The dependence of the mean effective temperature on $B$ for the dipole (dot-dash lines) and small-scale (solid lines) fields. The curves are shown for the accreted and iron envelopes and the internal temperature $T_{\mathrm{b}}=10^{7} \mathrm{~K}$ and $10^{8} \mathrm{~K}$ (marked near the curves).

energy crosses the magnetic Landau levels (see, e.g., Yakovlev \& Kaminker 1994; Ventura \& Potekhin 2001). The first oscillation at $\rho=\rho_{B}=7045 B_{12}^{3 / 2}(A / Z) \mathrm{g} \mathrm{cm}^{-3}$ is the strongest one (here $B_{12} \equiv B / 10^{12} \mathrm{G}$ and $A$ and $Z$ are the atomic weight and charge numbers); the classical (non-oscillating) magnetic electron conductivity is recovered at $\rho \gg \rho_{B}$. In Fig. 2, a decrease of $\bar{T}_{\mathrm{s}}^{\infty}$ first occurs in the classical regime, where the effect of the magnetic field amounts to a suppression of $\kappa_{\perp}$ in Eq. (3). With further increase of $B$, the field becomes quantizing in the sensitivity strip, leading to an increase of $\bar{T}_{\mathrm{s}}^{\infty}$. The increasing pieces of curves in Fig. 2 are wavy because of quantum oscillations of $\kappa_{\|}$, which are smoothed (but not entirely) by the integration of Eq. (3) over the sensitivity strip. 
In the case of the dipole magnetic field, the differences between the magnetic and nonmagnetic $T_{\mathrm{b}}\left(\bar{T}_{\mathrm{s}}\right)$-relations become appreciable only in a very strong magnetic field, $B \gtrsim 10^{14} \mathrm{G}$, and if the internal temperature is relatively low, $T_{\mathrm{b}} \lesssim 3 \times 10^{7} \mathrm{~K}$. In this case, $\bar{T}_{\mathrm{s}}$ is noticeably increased by the magnetic field. The increase of a mean effective temperature is however quite moderate in a comparison to the effect of such a strong field on the local temperature value: a significant increase of $T_{\mathrm{s}}$ at the magnetic pole and a sharp decrease at the equator (see, e.g., Potekhin \& Yakovlev 2001; Potekhin et al. 2003). For lower magnetic field and higher temperature, the difference in the magnetic geometry does not yield a considerable departure in the $T_{\mathrm{b}}\left(\bar{T}_{\mathrm{s}}\right)$-relation.

The difference in a magnetic configuration can manifest itself at $B \gtrsim 10^{10} \mathrm{G}$. The small-scale field, as a rule, results in a lower $\bar{T}_{\mathrm{s}}$ at a given $B$ compared to the dipole field. The difference in $\bar{T}_{\mathrm{s}}$ for the small-scale and dipole fields depends generally on $T_{\mathrm{b}}, B$, and the mass of the accreted light-element material (see Fig. 2), but never exceeds $20 \%$. However, this difference in $T_{\mathrm{s}}$ can result in a factor of $\sim 2$ in the luminosity. In all considered models, the uncertainty in a chemical composition of the envelope (light versus heavy elements) causes a much larger difference in $\bar{T}_{\mathrm{s}}$ than the uncertainty in the magnetic field geometry (dipole versus small-scale).

To estimate the effect of a small-scale magnetic field on the $T_{\mathrm{b}}\left(\bar{T}_{\mathrm{s}}\right)$-relation, one can use the fitting formulae derived for the dipole field (see the Appendix of Potekhin et al. 2003) where $\cos ^{2} \theta$ and $\sin ^{2} \theta$ should be replaced by $1 / 3$ and $2 / 3$, respectively. Such fit reproduces the present numerical results with an accuracy $\leq 10 \%$.

\section{Discussion}

We have considered the relation between the surface and internal temperature in neutron stars in the case where small scale magnetic fields near the surface are stronger than a large scale (e.g., dipole) field. Calculations show that the difference in the $T_{\mathrm{b}}\left(\bar{T}_{\mathrm{s}}\right)$-relation between the stars with small-scale and dipole fields is not very significant, although, generally, it should be taken into account if highly accurate thermal luminosity calculations are required. This can be important for the interpretation of future high-precision measurements of neutron star thermal radiation.

For both the small-scale and dipole fields, the departure from the thermal structure of a nonmagnetic neutron star is relatively small, if the typical field strength $B<10^{14} \mathrm{G}$. This is caused by the fact that the increase of thermal insulation near the region where the field lines are tangential is well compensated by the decrease of the insulation in the region where the field is normal to the surface. As seen from Fig. 2, for the dipole field this compensation is generally more efficient than for the small-scale field.

For a stronger field, an increase of the thermal conductivity along the field lines due to the magnetic quantization effects turns out to be so strong that it cannot be fully compensated by a decrease of the conductivity in the perpendicular direction.

Our results indicate that the geometry and strength of the magnetic field are likely unimportant for the average thermal structure of neutron stars, if the field strength is moderate $\left(B \lesssim 10^{14}\right)$. Therefore, a magnetic field of moderate strength can affect the neutron star cooling via Joule heating rather than via the $T_{\mathrm{b}}\left(\bar{T}_{\mathrm{s}}\right)$-relation. Joule heating is of particular importance at the late evolutionary stage and can maintain a relatively high surface temperature $\gtrsim 10^{5} \mathrm{~K}$ for a long time, comparable to the decay time of the magnetic field (Miralles et al. 1998).

Acknowledgements. The work of A.Y.P. and G.C. was partially supported by the CNRS French-Russian grant PICS 3202. The work of A.Y.P. was also supported in part by the RLSS grant 1115.2003 .2 and the RFBR grants 05-02-16245, 03-07-90200 and 05-02-22003.

\section{References}

Arons, J. 1993, ApJ, 408, 160

Arons, J. 2000, in Pulsar Astronomy - 2000 and beyond, Proc. IAU Coll. 177, ed. M. Kramer, N. Wex, \& R. Wielebinski (San Francisco: ASP), ASP Conf. Ser., 202, 449

Arons, J., \& Scharlemann, E. T. 1979, ApJ, 231, 854

Becker, W., Swartz, D. A., Pavlov, G. G., et al. 2003, ApJ, 594, 798

Bonanno, A., Rezzolla, L., \& Urpin, V. 2003, A\&A, 410, L33

Bonanno, A., Urpin, V., \& Belvedere, G. 2005, A\&A, 440, 199

Geppert, U., Rheinhardt, M., \& Gil, J. 2003, A\&A, 412, L33

Gil, J., \& Melikidze, G. 2002, ApJ, 577, 909

Gil, J., \& Mitra, D. 2001, ApJ, 550, 383

Gil, J., \& Sendyk, M. 2000, ApJ, 541, 351

Greenstein, G., \& Hartke, G. J. 1983, ApJ, 271, 283

Gudmundsson, E. H., Pethick, C. J., \& Epstein, R. I. 1983, ApJ, 272, 286

Hernquist, L. 1985, MNRAS, 213, 313

Heyl, J. S., \& Hernquist, L. 1998, MNRAS, 300, 599

Mineo, T., Cusumano, G., Massaro, E., Becker, W., \& Nicastro, L. 2004, A\&A, 423, 1045

Miralles, J., Urpin, V., \& Konenkov, D. 1998, ApJ, 503, 368

Nomoto, K., \& Tsuruta, S. 1987, ApJ, 312, 711

Page, D. 1995, ApJ, 442, 273

Page, D. 1997, ApJ, 479, L43

Page, D. 1998, in The Many Faces of Neutron Stars, ed. R. Buccheri, J. van Paradijs, \& M. A. Alpar (Dordrecht: Kluwer), 539

Pethick, C. J. 1992, Rev. Mod. Phys., 64, 1133

Potekhin, A. Y., \& Chabrier, G. 2004, ApJ, 600, 317

Potekhin, A. Y., \& Yakovlev, D. G. 2001, A\&A, 374, 213

Potekhin, A. Y., Chabrier, G., \& Yakovlev, D. G. 1997, A\&A, 323, 415

Potekhin, A. Y., Yakovlev, D. G., Chabrier, G., \& Gnedin, O.Y. 2003, ApJ, 594, 404

Ruderman, M. 1991, ApJ, 382, 587

Ruderman, M., Zhu, T., \& Chen, K. 1998, ApJ, 492, 267

Schaaf, M. E. 1990, A\&A, 227, 61

Thompson, C., \& Duncan, R. C. 1993, ApJ, 408, 194

Thorne, K. S. 1977, ApJ 212, 825

Urpin, V., \& Gil, J. 2004, A\&A, 415, 305

Van Riper, K. A. 1988, ApJ, 329, 339

Ventura, J., \& Potekhin, A. Y. 2001, in NATO Science Ser. C, 567, The Neutron Star - Black Hole Connection, ed. C. Kouveliotou, J. Ventura, \& E. P. J. van den Heuvel (Dordrecht: Kluwer), 393

Yakovlev, D. G., \& Kaminker, A. D. 1994, in The Equation of State in Astrophysics, ed. G. Chabrier, \& E. Schatzman (Cambridge: Cambridge University Press), 214

Yakovlev, D. G., \& Pethick, C. J. 2004, ARA\&A, 42, 169 